\title{
EXISTENCE AND REGULARITY OF MINIMAL SURFACES ON RIEMANNIAN MANIFOLDS
}

\author{
BY JON T. PITTS ${ }^{1}$
}

Communicated by Shing S. Chern, January 9, 1976

This is a research announcement the full details of which appear in [PJ1] and [PJ2]. We study the existence and regularity of $k$ dimensional minimal surfaces on smooth riemannian manifolds.

Suppose $M$ is a smooth compact riemannian manifold of dimension $n$, and $k \leqslant n$ is a positive integer. It is a problem of long standing to show that $M$ supports a regular closed minimal submanifold of dimension $k$. Until now the only general existence theorem known which required no additional hypothesis on $M$ applied when $k=1$. In this case the variational methods of M. Morse imply the existence of closed geodesics on $M$, possibly with self-intersections. Results established for $k \geqslant 2$ have required additional geometric or topological hypotheses on $M$. For example, if $k=n-1 \leqslant 6$, then the support of every nonzero homologically area minimizing integral cycle is a smooth closed embedded $k$ dimensional submanifold, a fact established by the methods of [FH, Chapter 5]. Thus one settles the existence problem affirmatively in these dimensions whenever the $k$ dimensional homology group of $M$ with coefficients in the integers does not vanish. Another example is the theorem of Lawson [LHB] that $M=$ $\mathbf{S}^{3}$ supports closed two dimensional minimal submanifolds of arbitrarily high genus.

There are few results known in the general case, where $M, k$, and $n$ are arbitrary. We announce an application of variational methods similar to those of Almgren [AF] and Morse to show that $M$ supports a nonzero stationary $k$ dimensional integral varifold which at each point of $M$ is almost minimizing in all sufficiently small annular neighborhoods of that point. Almost minimizing varifolds are principal objects of our investigation. Intuitively one considers an almost minimizing varifold to be one which may be approximated arbitrarily closely by integral currents, which are themselves very nearly locally minimizing. A varifold which is only stationary and integral has in general essential singularities, possibly of positive measure. If in addition the varifold is almost minimizing, then it possesses strong local stability properties which yield estimates on the

AMS (MOS) subject classifications (1970). Primary 49F10, 49F20, 49F22.

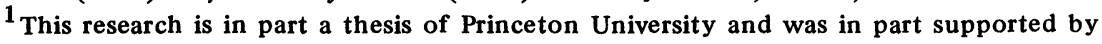
National Science Foundation Grant MPS 75-06984. 
singular sets. In particular, when $k=2$ and $n=3$, these estimates imply that the singular set is empty, and we conclude, for example:

THEOREM. Every smooth compact three dimensional riemannian manifold contains a nonempty closed embedded two dimensional minimal submanifold without boundary.

Our methods apply to prove a general theorem analogous to the well-known results of Morse-Tompkins [MT] and Shiffman [SM] on existence of unstable minimal surfaces which span curves bounding two locally area minimizing surfaces. In particular, suppose $C$ is a one dimensional integral cycle in $\mathbf{R}^{3}, T_{1}$ and $T_{2}$ are two dimensional integral currents, $\partial T_{1}=\partial T_{2}=C$, and $T_{1}$ and $T_{2}$ locally minimize area among all integral currents with boundary $C$. Then there exists a two dimensional integral varifold $V$ such that $V$ is stationary with respect to deformations with compact support in $\mathbf{R}^{\mathbf{3}} \sim \operatorname{spt} C, \infty>\|V\|\left(R^{\mathbf{3}}\right)>$ $\max \left\{\mathbf{M}\left(T_{1}\right), \mathbf{M}\left(T_{2}\right)\right\}$, spt $C \subset \operatorname{spt}\|V\|$, and spt $\|V\| \sim \operatorname{spt} C$ is a two dimensional real analytic submanifold of $\mathbf{R}^{3}$.

For example, suppose $C$ consists of two parallel circles in $\mathbf{R}^{3}$ oriented oppositely. If the circles are sufficiently close, and if we choose $T_{1}$ to be two parallel discs suitably oriented and $T_{2}$ to be the area minimizing catenoid spanning $C$, then our methods imply the existence of a second, generally unstable, catenoid spanning $C$ lying between $T_{1}$ and $T_{2}$. This and other simple examples illustrate that unlike the situation in [MT] and [SM], there are no topological restrictions on the spanning surfaces. In particular, while it is true that spt $T_{1} \sim$ spt $C$ and spt $T_{2} \sim$ spt $C$ are two dimensional real analytic submanifolds of $\mathbf{R}^{3}$ [FH, Chapter 5], it may happen that no two of spt $T_{1} \sim \operatorname{spt} C$, spt $T_{2} \sim \operatorname{spt} C$, and spt $\|V\| \sim \operatorname{spt} C$ have the same topological type.

\section{REFERENCES}

[AF] F. J. Almgren, Jr., The theory of varifolds. Mimeographed notes, Princeton

[FH] H. Federer, Geometric measure theory, Die Grundlehren der math. Wissenschaften, Band 153, Springer-Verlag, New York, 1969. MR 41 \#1976.

[LHB] H. B. Lawson, Jr., The global behavior of minimal surfaces in $S^{n}$, Ann. of Math. (2) 92 (1970), 224-237. MR 42 \#169.

[MT] M. Morse and C. Tompkins, The existence of minimal surfaces of general critical types, Ann. of Math. (2) 40 (1939), 443-472.

[PJ1] J. Pitts, Existence of minimal surfaces on riemannian manifolds I: Almost minimizing varifolds (preprint).

[PJ2] - Existence of minimal surfaces on riemannian manifolds II: Regular surfaces in three dimensional manifolds (preprint).

[SM] M. Shiffman, The plateau problem for non-relative minima, Ann. of Math. (2) 40 (1939), 834-854. MR 1, 79.

DEPARTMENT OF MATHEMATICS, UNIVERSITY OF ROCHESTER, ROCHESTER, NEW YORK 14627 